\title{
DEEP ENSEMbLE LEARNING-BASED SMART TEACHING
}

\author{
(1) Rajdeep Chatterjee ${ }^{*}$ \\ School of Computer Engineering \\ KIIT Deemed to be University \\ Bhubaneswar-751024, Odisha, India \\ cse.rajdeep@gmail.com
}

\author{
Rohit Halder \\ School of Computer Engineering \\ KIIT Deemed to be University \\ Bhubaneswar-751024, Odisha, India \\ rhaldar9@gmail.com
}

\begin{abstract}
In the traditional system, a teacher observes its students' attention levels from his/her experience. To date, there is no system that automatically tracks the attention level of the students in a class in real-time (that is while the lecturer delivering his/her lectures or student watching tutorials). This paper aims at improving the lecture delivery mechanism in real-time in a classroom. On the other hand, our proposed system periodically will not only monitor the learning behaviour of the whole class but also track the attentiveness of each student. The proposed system is not meant to identify the non-attentive students and punish them. Rather contrary to the punishment based mechanism, it introduces a counseling based mechanism. This deep learning-based real-time face monitoring system will allow lecturers to improvise his/her delivery either through bringing diversity in the class contents or personal care to those non-attentive students. The concept of deep ensemble learning has been used with convolutional neural networks to predict the percentage of openness of eyes. Separately, the positive facial postures of a student are also recognized using our model of convolutional neural network. Finally, the net learning behaviour of the student has been computed by a weighted average of these two features (that are, eyes openness and facial postures).
\end{abstract}

Keywords Attention $\cdot$ Deep learning $\cdot$ Digital classroom $\cdot$ Learning behaviour $\cdot$ Smart teaching

\section{Introduction}

Deep learning applications have not been implemented in the education domain at a satisfactory level. It has a tremendous scope for improving the traditional teaching-learning experiences in the class rooms. Teachers find it difficult to monitor the attention level of students in their classes in real-time. Traditionally, they believe in their experience, but experience does not guarantee an accurate monitoring. Thus, they may be unable to provide corrective measures to improve the attention level of the students during an oral lecture. There are three major reasons why monitoring of the students' attention level is important:

1. If the average attention of the whole class is high, then the lecture delivery on a particular topic will be easy and smooth. This will be beneficial for the students as a whole.

2. If the students are attentive, then the lecturer can concentrate on delivery rather than controlling the class or maintaining the overall discipline.

3. Suppose a student or a group of students intent(s) to listen to the lecture but in due course of the lecture loss the attention level. An intelligent attention monitoring system could help them to re-concentrate or the teacher/instructor can identify them and counsel.

\footnotetext{
${ }^{*}$ Corresponding author.
} 
Attention and posture has always been the essential parameter to analyze the contentment level of students while attending the lectures. In this paper, a lightweight computational model has been introduced for better analysis of the attention level and the satisfaction level of a student in real time, while attending a lecture. Our approach is quite simple, we have proposed a smart classroom, which helps us calculate the postural behaviours and percentage of opening and closing of eyes in real-time, which indeed gives the overall attention level for a duration of time (we call them sessions). The benefits of such real time feedback, includes:

(a) If the attention of the entire lecture room falls as shown in Fig 1 , the instructor must change his way of teaching by making the lecture content more appealing to the students.

(b) If the attention of a particular few students fall short as shown in Fig. 2, they can self-introspect as to why they are not able to pay proper attention, and ask the instructor for help.

This is also an initial step to the augmented teaching-learning system which will enhance the quality of the overall classroom environment through intelligent decisions and feed-back mechanisms.

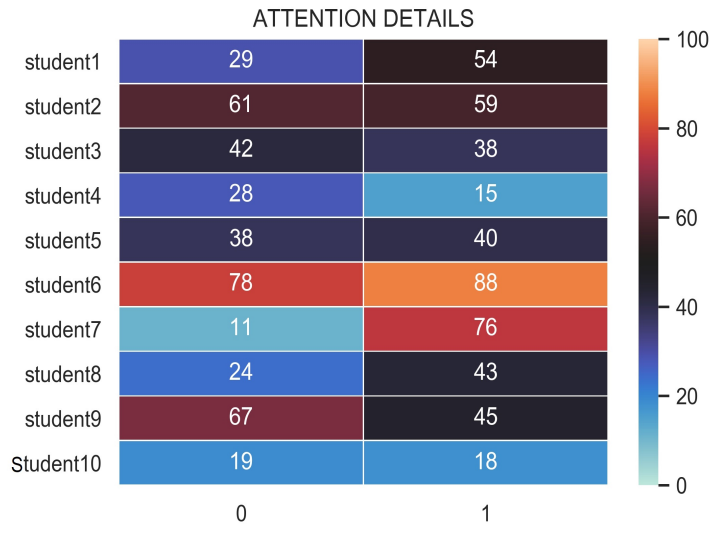

Figure 1: Global poor attention.

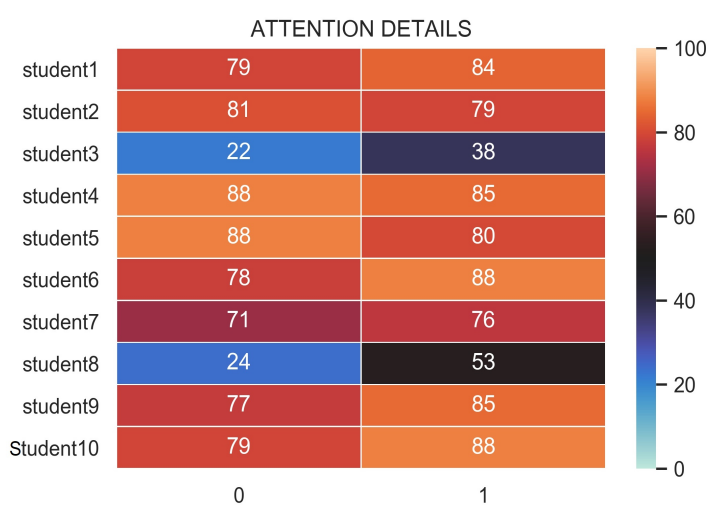

Figure 2: Local poor attention.

The rest of the paper has been organized as follows. In Section 2 , previous approaches regarding attention or posture level detection have been briefly discussed. This is followed by Section 3, a detailed overview of our proposed approach and the used models have been given. This is followed by Section 4 where brief description of the datasets, associated image preprocessing and the model architecture are explained. The results analysis and observations of our experiments have been discussed in 5. Finally, the paper has been concluded in Section 6 .

\section{Related Works}

The research on attention and memory analysis has begun its globe-trotting with the study of Electroencephalogram (EEG), electrooculogram (EOG), electrocardiogram (ECG). The variation in skin temperature and electrodermal activity, have been initially used to predict the attention level of the subjects [1]. P300-based brain-computer interface (BCI) has been also used to analyze the attention and memory process [2]. But the process by which these sensations or brain waves are recorded unfavorable for students to have the instruments attached to their body while attending the lectures [3]. It is also very much prone to the different types of biological or external artifacts.

With the evolution of computer vision, algorithms based on pattern analysis [4], max margin face detection [5], fuzzy growth [6], feature extraction including relative structure and positioning of facial components with cranial structures, cerebral size, naso-olfactory adaptations to brain size [7] which certainly make the analysis of attention feasible and easier to implement. The research has not been limited to a face detection or eyes openness only. Facial postures, 
body gestures have also taken the ground to analyze a person's behaviour using the temporal features as well as the spacial features [8].

Literature tell us about researches on the smart learning classroom including the impact of the teachers position on students performance [9], practice of in-classroom manual feedback of the students [10], smart techniques and frameworks for the students with disabilities [11]. These techniques are growing now a days to completely understand the behaviour of the students while attending the lectures and take corrective measures as per their requirements. This intelligent improvements in classroom certainly help the students to get a clear understanding about their courses.

The concept of deep ensemble learning has been introduced to identify and differentiate between multiple audio signals [12]. Later, the authors has proposed similar approaches for video classification [13]. We have merged few of the previous approaches $(\mathrm{CNN}+$ Deep ensemble learning), to put forward a detailed analysis on the learning behaviour of a student subject in a lecture room environment, using the attention (eyes openness) analysis and facial posture analysis as prime metrics.

\section{Proposed Approach}

The proposed approach to monitoring and analyze the contentment of the students learning behaviour has two aspects: Firstly, the percentage of eyes openness of a person suggests wakefulness. It is necessary to perform any reasonable cognitive task or interaction with the outside world (here, it is classroom); Secondly, the facial postures (expressions) are another feature to track the positive or curious mindset of a person. In this paper, these two said features have been combined to determine the net learning behaviour of a student.

\subsection{Attention Analysis}

The attention analysis mechanism uses a concept of ensemble deep learning, where three independent CNN [14] models, trained on different datasets are used to predict the openness of the eyes [15], while the students are attending their lectures, by means of the front camera of the laptops or PC webcams. Majority voting combining technique has been used to get the final result from the ensemble (refer Eq. 11).

$$
\operatorname{Majority}\left(W_{1}, W_{2}, W_{3}\right)=W_{i}
$$

Where $W_{1}, W_{2}$ and $W_{3}$ are the predicted results from model-1, model-2 and model-3 respectively. The weighted average of $W_{i}$ and $W_{j}$ gives the net $\%$ of openness of eyes. An equal-weighted (0.5) sum has been used to calculate the final outcome using Eq. 2

$$
W=\left(0.5 * W_{i}\right)+\left(0.5 * W_{j}\right)
$$

Any student, not attending the session properly or found sleeping partially or fully or talking with classmates, will be marked with 0 or significantly low attention level respectively. The attentive students will have significantly high attention level.

\subsection{Posture Analysis}

Our approach uses only spatial features to detect the facial posture and classify, whether it is positive, negative or neutral. Temporal features have not been used in the entire system to make it computationally light. Different facial expressions convey different levels of satisfaction. If a student is found yawning, he/she is probably sleepy and is not having enough interest in attending the lecture. Or if a student is attending the lecture with eyes wide open and leaning a little forward, it indicates his eagerness to learn from the lecture and so on. A facial posture level indicates the eagerness of a student to learn from the lectures, a global poor posture level denotes a bad choice of lecturing technique, whereas a local poor posture level suggests the problem of few particular students to understand the lecture. 


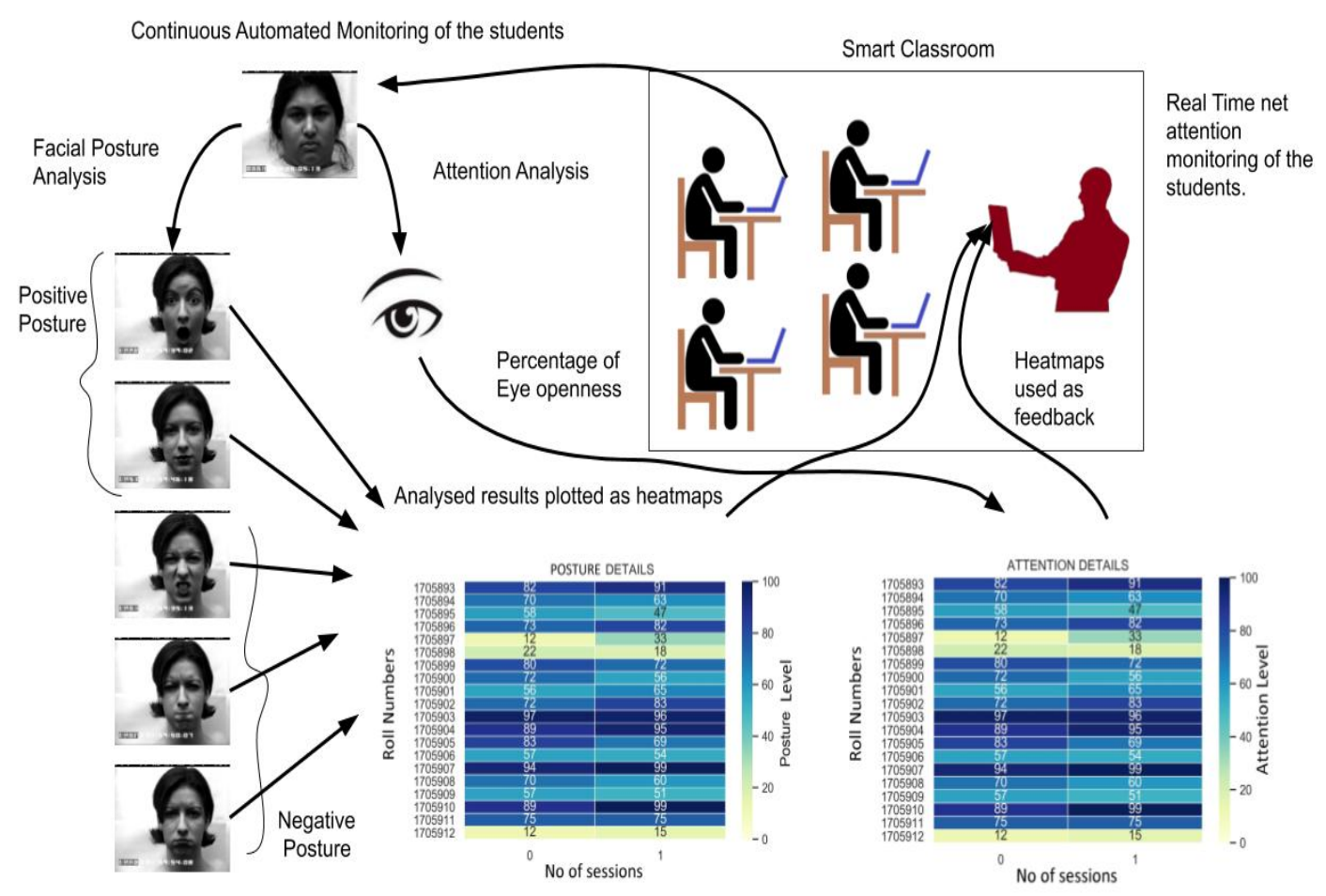

Figure 3: Overview of our research.

The overview of our proposed approach has been shown in Fig. 3. It depicts a smart classroom where students are watching either live stream of a lecture or on-line tutorial video through their individual web-camera mounted PCs or laptops. Our proposed attention and facial posture monitoring techniques are being executed simultaneously in each session (that is, equal temporal divisions of the whole lecture). Eventually, the results of the attention and posture analysis have been combined by taking a mean to obtain the final net learning behaviour of the students.

\section{Experimental Setup}

\subsection{Datasets}

The effectiveness of each of our model has been validated using the standard datasets. First, we have used the following datasets to examine the accuracy of our individual used models. Then, we have used our own datasets to establish the proposed technique for the smart education system.

\subsubsection{The Extended Cohn-Kanade Dataset (CK+)}

$\mathrm{CK}+$ contains 593 video sequences on both posed and non-posed emotions. The age range of its 123 subjects ranges from 18 to 30 years. The majority of the subjects are females. Image sequences may be analyzed for both action units and prototypic emotions. The images that has been used here has a pixel resolutions of $640 \times 480$ and $640 \times 490$ with 8-bit precision for gray-scale values [16].

\subsubsection{Dataset B Eye Images}

The dataset was released in 2013 by PARNEC 2 (PAttern Recognition and NEural Computing) group is affiliated with the College of Computer Science and Technology, Nanjing University of Aeronautics and Astronautics, China. The dataset contains the open and closed eye images for the left and right eyes. The dataset contains 1232 open eye images,

\footnotetext{
${ }^{2}$ http://parnec.nuaa.edu.cn/
} 
for left and right eyes. Whereas only 1193 closed eye images, for left and right eyes. Hence the total number of 4850 subject images, each of which is $24 \times 24$ [17].

\subsubsection{Dataset B Facial Images}

The dataset was also released in 2013 by PARNEC (PAttern Recognition and NEural Computing) group is affiliated with the College of Computer Science and Technology, Nanjing University of Aeronautics and Astronautics, China. The dataset contains the images of the face along with the open eye or closed eye. The dataset contains 2464 subject images each of which is $100 \times 100$ [18].

\subsubsection{Our Experimental Dataset}

The above-proposed approach for determining learning bahivour has been practically tested on 10 healthy male students with $18-25$ year age group. The proposed models are running in the background while the student volunteers have been asked to watch two separate videos with a 10 minutes refreshment break. Each of the videos has a duration of 15 minutes. The whole tutorial video is partitioned into 5 of 3 minutes session. The brief overview of the experiments have been given in Table 1

\subsubsection{Experiment-I}

The students have been asked about their domain of interests, which included movie-making, bookkeeping, motivational talks, latest gaming videos and many more. The videos of their respective interests have been shown to them. The behaviour of the students has been monitored using our proposed approach. It must be noted that the given videos for our 10 student volunteers vary due to their interest in different areas.

\subsubsection{Experiment-II}

Never seen videos have been given to the students from their non-interested domain (here, it is a "software engineering" topic). Here, it needs to be clearly understood that the shown video is kept the same for all the student volunteers as they willfully accept that they do not have an interest in the said topic.

Table 1: Brief overview of our experiments

\begin{tabular}{|l|l|l|}
\hline $\begin{array}{l}\text { Experiment } \\
\text { Type }\end{array}$ & Acronym & Brief Description \\
\hline Experiment-I & Exp-I & $\begin{array}{l}\text { Never seen video lec- } \\
\text { ture from domain of } \\
\text { student's interest. }\end{array}$ \\
\hline Experiment-II & Exp-II & $\begin{array}{l}\text { Never seen video lec- } \\
\text { ture from un-known } \\
\text { (non-interested) do- } \\
\text { main. }\end{array}$ \\
\hline
\end{tabular}

\subsection{Preprocessing}

A $\langle a, x, y, z\rangle$ tensor means a frames are used to train the model, the height and width of the frame is $\mathbf{x}$ and $\mathbf{y}$ pixels respectively and the frame is a $\mathbf{z}$-dimensional matrix or simply a colored image for $z=3$. For the detection of faces and the openness of the eyes, the frames have been resized to $30 \times 30$. Hence, the resulting tensor is $\langle a, 30,30,3\rangle$. For the detection of posture level, the frames have been resized to $100 \times 100$. Hence the resulting tensor for posture detection is $\langle a, 100,100,3\rangle$. All the frames have been processed in BRG color channels. The class labels associated with the tensors respectively are also fed while training the model.

\subsection{Model Architecture}

The entire system consists of 6 models based on deep learning and 2 models based on haar cascades: 


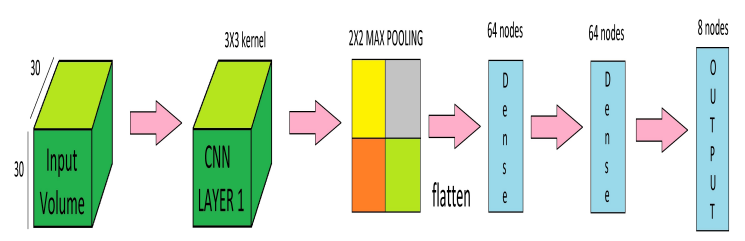

FACEDEEECTON MODEL

Figure 4: Face detection model

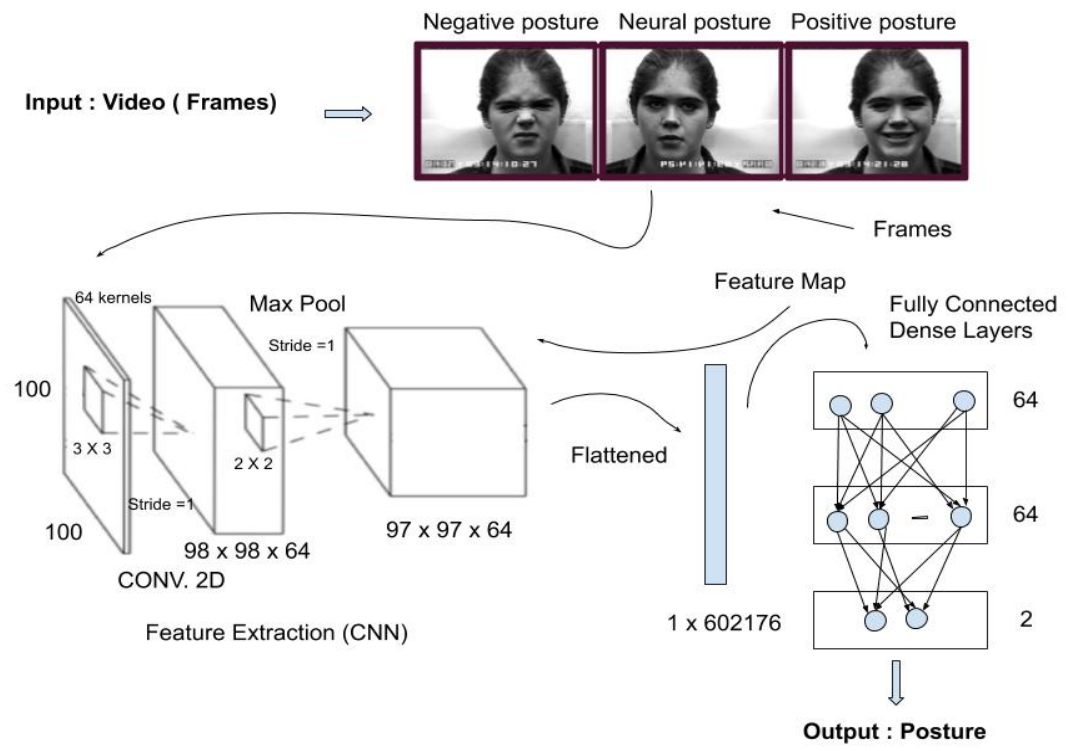

Figure 5: Posture detection model

1. A deep-learning model (CNN + Dense Layers) for detecting students' faces as shown in Fig 4

2. A deep-learning model (CNN + Dense Layers) for detecting (students) posture as shown in Fig. 5 .

3. A dense deep-learning model (CNN + Dense Layers) for detecting (students) percentage of openness of eyes.

4. An ensemble deep-learning model (CNN + Dense Layers) [19] for detecting the percentage of eyes openness. The ensemble model consists of three normal deep learning models (CNN + Dense Layers), shown in Fig. 66 which works together as a whole. The probability of error by a single model is high, but a council of models is more efficient with a low probability of error.

The mechanism of detecting the openness of eyes by using multiple models is shown in Fig. 7

We have converted the probability of an open eye into a percentage, to get the attention levels. Like a completely open eye is most probable to give a 100 percentage attention level, a half-open eye to show a 50 percentage attention level and a closed eye to show a 0 percentage attention level. Similarly, positive posture, that is, willingness to study 


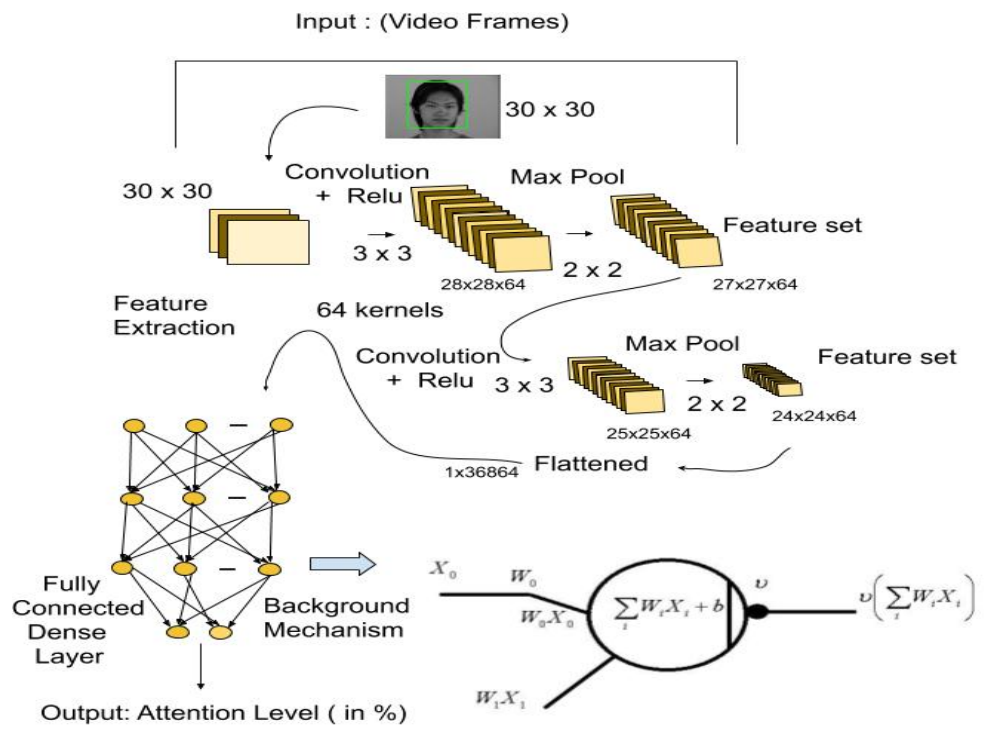

Figure 6: Single attention detection model used in the ensemble

is denoted by 100 percent posture level, a negative posture, that is, unwillingness to study is denoted by 0 percent posture level, and a neutral posture level is unpredictable is denoted by 50 percent posture level.

\subsection{Training Methodology}

All the models have been build and trained from scratch for 80 epochs. The model has been trained using the Adam optimizer due to its computational efficiency, invariance to the diagonal rescaling of gradients, low memory requirement, and its capacity to handle quite a high volume of training data. The learning rate has been kept 0.001 , the exponential decay rate for the $1^{\text {st }}$ moment estimates is fixed at 0.9 whereas the exponential decay rate for the $2^{\text {nd }}$ moment estimates are kept at 0.999 . The loss function that has been used in this paper is "sparse categorical crossentropy". As we have dealt with multiple classes in our approach, we have used " 0 or 1 " as class labels, instead of one-hot encoding. The batch size of 5 samples at an instant has been kept constant while training. The dataset has been divided into a $9: 1$ ratio, for training and validating purposes.

\subsection{System Configuration}

Intel Core $i 5,7^{\text {th }}$ generation HP Pavilion x360 laptop with 8GB RAM and Windows 10 Professional operating system. For coding, Python 3.6 is used with Jupyter Notebook in Anaconda environment.

\section{Results and Analysis}

The following subsections give a detailed analysis of the proposed approach for determining the students learning pattern.

\subsection{Attention Detection}

The Figs. 8, 9, 10 and 11 refer to the test (or validation) accuracies, while validating our different models. The Attention Detection model or model-4 shown in Fig. 7 has obtained the maximum accuracy during the epochs 30 to 40 , giving a mean validation accuracy to $96.22 \%$. Similarly, the other models that make up the deep ensemble model 


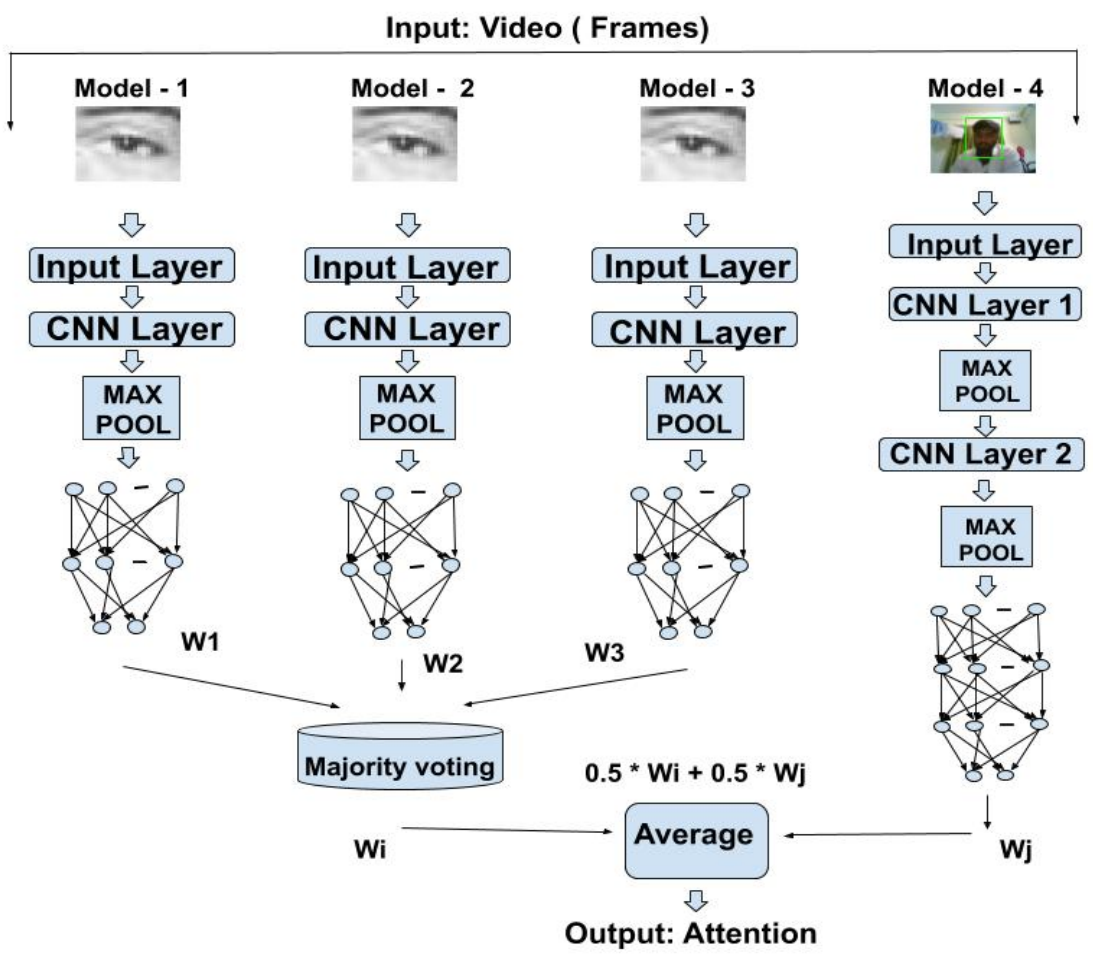

Figure 7: Attention detection system

have the validation accuracy of $99.35 \%, 98.35 \%$ and $97.78 \%$ respectively.
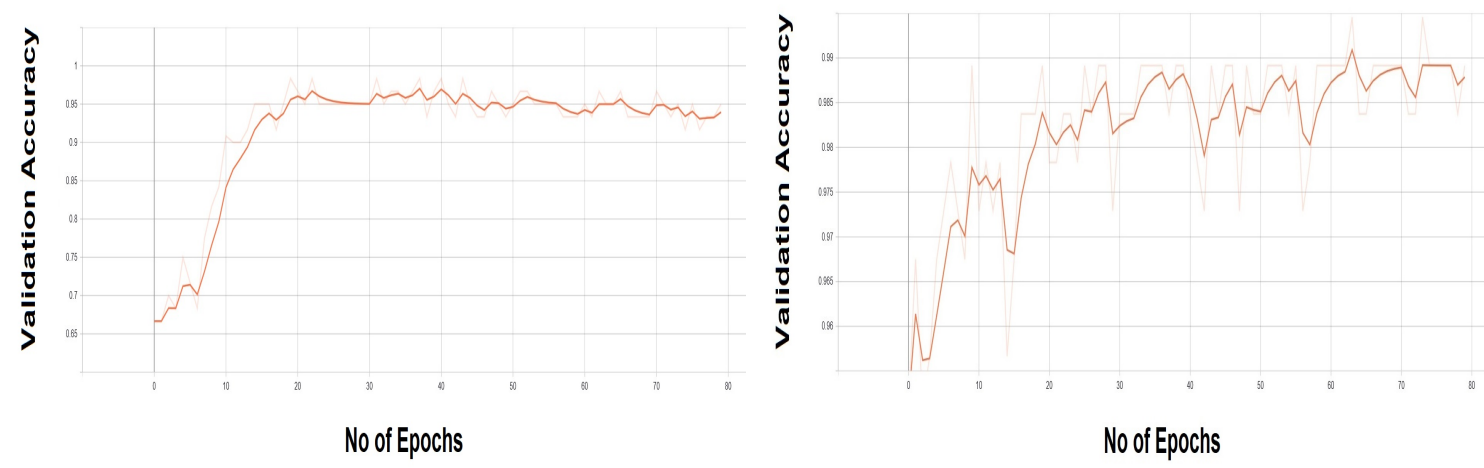

Figure 8: Attention detection validation accuracy Figure 9: Ensemble model-1 validation accuracy graphs graphs 


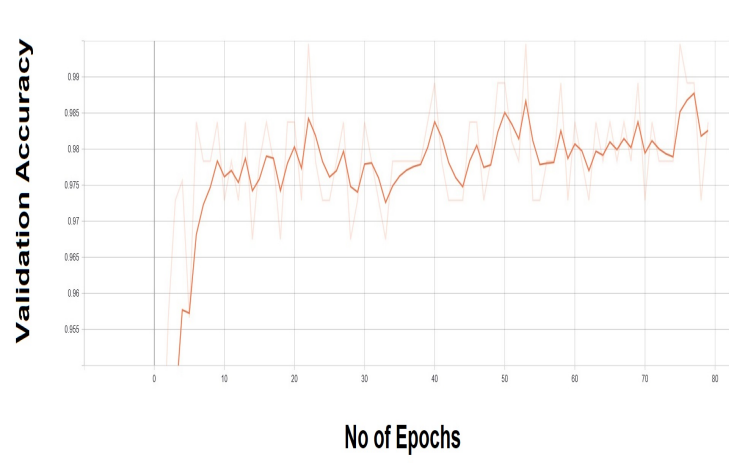

No of Epochs

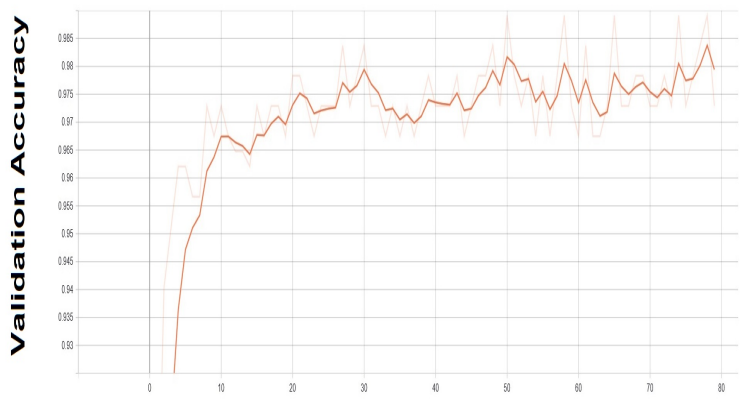

No of Epochs

Figure 10: Ensemble model-2 validation accuracy Figure 11: Ensemble model-3 validation accuracy graphs graphs

\subsection{Posture Detection}

The validation accuracy graph for posture detection is shown in Fig. 12. From the graph, we can see that the maximum accuracy was obtained in epochs ranging from 35 to 45 . Taking a mean of the validation accuracies from $35^{\text {th }}$ epoch to $45^{\text {th }}$ epoch, we get a net accuracy of $97.98 \%$.

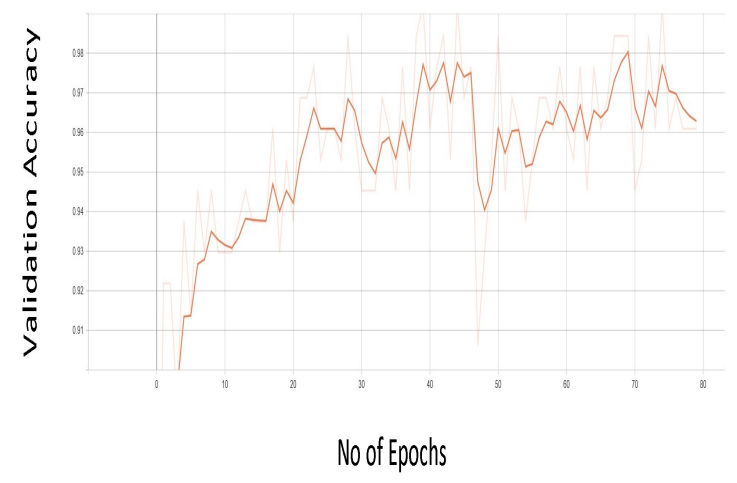

Figure 12: Posture detection validation accuracy graph

\subsection{Observation from Experiment-I}

Most of the subjects have good attention (that is, eye openness) and positive facial postures levels. Another noticeable observation includes that the subjects start with low learning behaviour which gradually increases over time. It suggests two aspects of our study: (a) human mind takes time to become attentive, and (b) the continuous feedback (that is, the session-wise heat-maps of net learning behaviour of the students) helps the instructor to take corrective measures. Therefore, this proposed method validates our proposal that deep learning-based attention monitoring can enhance the overall teaching-learning behaviour of the students in a class or a student while watching an on-line tutorial video. 


\begin{tabular}{r|c|c|c|c|c|c} 
& \multicolumn{7}{c}{ ATTENTION DETAILS } & \multicolumn{1}{c}{-100} \\
student1 & 79 & 84 & 81 & 79 & 82 & \\
\cline { 2 - 6 } student2 & 68 & 88 & 85 & 88 & 80 \\
student3 & 78 & 88 & 71 & 76 & 84 & -80 \\
student4 & 73 & 77 & 85 & 84 & 80 \\
student5 & 89 & 85 & 87 & 83 & 88 \\
student6 & 80 & 83 & 78 & 83 & 87 \\
\hline student7 & 76 & 81 & 84 & 79 & 82 \\
student8 & 76 & 73 & 75 & 81 & 77 \\
student9 & 77 & 79 & 84 & 81 & 89 \\
\hline Student10 & 82 & 79 & 65 & 78 & 84 & -20 \\
& 0 & 1 & 2 & 3 & 4 & -0
\end{tabular}

Figure 13: Obtained attention level from Exp-I

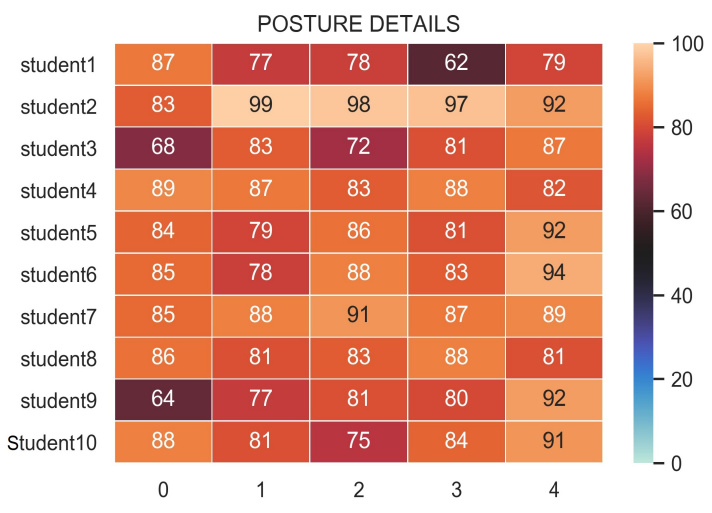

Figure 14: Obtained facial posture from Exp-I

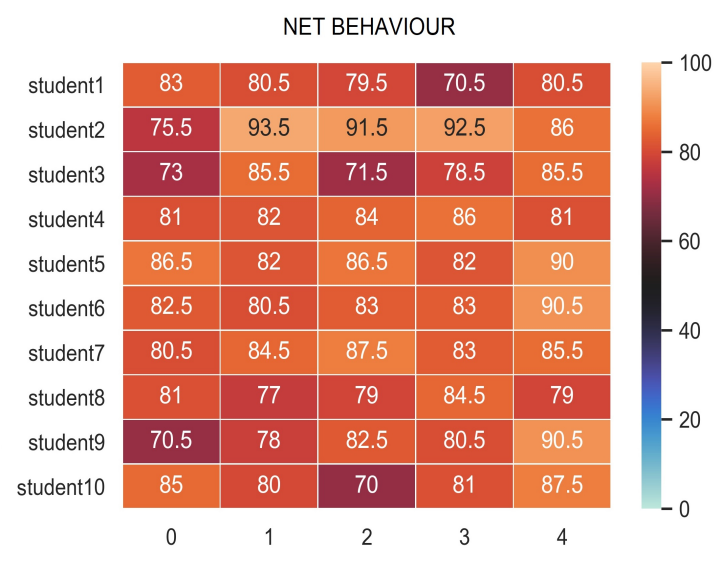

Figure 15: Obtained students' net behaviour from Exp-I

\subsection{Observation from Experiment-II}

Only 3 students (students 5,8 and 10) are found to have a stable attention level for all the sessions in the experiment-II. The net learning behaviour for the rest of the subjects has been further decreased over time. The psychological aspect of this observation is that our human brain first tries to understand any topic if the content is related and relevant, the learning process becomes faster. Otherwise, the learning behaviour slowly degrades over time even if the instructor counsels the students to pay proper attention.

The experimental results are shown using color heat-maps where the $x$ axis denotes sessions (time) and the $y$ axis indicates students (here, total 10 students). A separate color bar is also given to display the confidence level in a range of $0-100$. The color of each cell of the heat-map becomes lighter orange if confidence goes up otherwise it becomes lighter blue. The black shed is displayed when the confidence level is around $50 \%$.

The details of the observed results are shown in Figs. 13 and 14 for the experiment-I (Exp-I), 16 and 17 for the experiment-II (Exp-II) using color heat-maps. The net students learning behaviour have been given in Figs. 15 and 18 for interested and non-interested tutorials respectively. The confidence levels are being depicted in $\%$ for all the heat-maps shown in this paper. Finally, a sample result of our study has been displayed in Fig. 19. It shows each of the student's face cropped from the video (captured using the system camera) using our deep learning model. Then, these faces feed to the attention detection in one hand and the posture detection on the other hand. The obtained results for 
each student are also given in 19 for an easy understanding of our proposed approach. The student volunteers have given their consents to publish their photos in this paper.

\begin{tabular}{l|c|c|c|c|c|c} 
& \multicolumn{7}{c}{ ATTENTION DETAILS } & \multicolumn{1}{c}{-100} \\
\cline { 2 - 6 } student1 & 55 & 39 & 65 & 34 & 37 & -80 \\
\cline { 2 - 6 } student2 & 80 & 72 & 65 & 56 & 29 \\
student3 & 59 & 57 & 61 & 53 & 43 & -60 \\
student4 & 63 & 57 & 55 & 54 & 50 \\
student5 & 67 & 71 & 62 & 78 & 83 \\
student6 & 60 & 39 & 68 & 43 & 47 \\
student7 & 67 & 72 & 56 & 42 & 47 & -40 \\
student8 & 66 & 73 & 75 & 81 & 87 \\
student9 & 56 & 68 & 27 & 50 & 34 \\
\hline Student10 & 82 & 76 & 83 & 81 & 85 & -20 \\
& 0 & 1 & 2 & 3 & 4 &
\end{tabular}

Figure 16: Obtained attention level from Exp-II

\begin{tabular}{l|c|c|c|c|c|c} 
& \multicolumn{7}{c}{ POSTURE DETAILS } & \multicolumn{1}{c}{-100} \\
\cline { 2 - 6 } student1 & 62 & 43 & 49 & 46 & 51 & -80 \\
\cline { 1 - 6 } student2 & 87 & 68 & 54 & 46 & 23 \\
student3 & 87 & 64 & 72 & 64 & 54 \\
student4 & 74 & 71 & 62 & 67 & 63 \\
student5 & 77 & 83 & 78 & 84 & 89 \\
\hline student6 & 55 & 38 & 88 & 53 & 54 \\
\hline student7 & 68 & 71 & 45 & 57 & 55 & -40 \\
student8 & 72 & 75 & 69 & 85 & 94 \\
student9 & 54 & 61 & 25 & 32 & 27 \\
\hline Student10 & 77 & 75 & 86 & 79 & 91 & -20 \\
& 0 & 1 & 2 & 3 & 4 &
\end{tabular}

Figure 17: Obtained facial posture from Exp-II

\begin{tabular}{|c|c|c|c|c|c|}
\hline \multicolumn{6}{|c|}{ NET BEHAVIOUR } \\
\hline student1 & 58.5 & 41 & 57 & 40 & 44 \\
\hline student2 & 83.5 & 70 & 59.5 & 51 & 26 \\
\hline student3 & 73 & 60.5 & 66.5 & 58.5 & 48.5 \\
\hline student4 & 68.5 & 64 & 58.5 & 60.5 & 56.5 \\
\hline student5 & 72 & 77 & 70 & 81 & 86 \\
\hline student6 & 57.5 & 38.5 & 78 & 48 & 50.5 \\
\hline student7 & 67.5 & 71.5 & 50.5 & 49.5 & 51 \\
\hline student8 & 69 & 74 & 72 & 83 & 90.5 \\
\hline student9 & 55 & 64.5 & 26 & 41 & 30.5 \\
\hline \multirow[t]{2}{*}{ student10 } & 79.5 & 75.5 & 84.5 & 80 & 88 \\
\hline & 0 & 1 & 2 & 3 & 4 \\
\hline
\end{tabular}

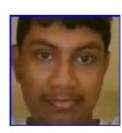

Subject 1

Known Video

Attention:81

Posture:76.6
Net -
Behaviour:78.8

Behaviour:78.8

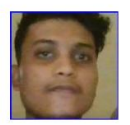

Subject 6 Attention::86. Posture: 85.6 Net-
Behaviour:86

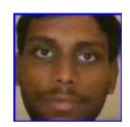

Subject 2 Known Video
Attention:81.8 Post -
Net Behaviour:87.8

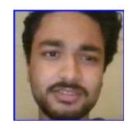

Subject 7
Known Video Attention:80.4 Posture:88 Net -
Behaviour:84.2

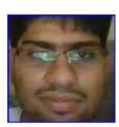

Subject 2

Known Video

Attention:79.4

Posture:

Net-
Behaviour:78.8

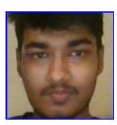

Subject 8

Attention:76.4
Posture:83.8

Net-
Behaviour:80.1

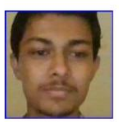

Subject 4

Known Video

Attention:79.8

Posture: 85.8

Behaviour:82.8

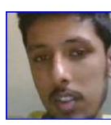

Subject 9

Known Video

Posture:78.8

Net -
Behaviour:80.4

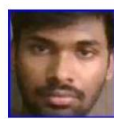

Subject 5

Known Video

Attention: 79.8

Behaviour:82.

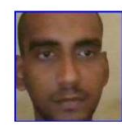

Subject 10
Known Video

Attention:77.6

Attention:77.6
Posture: 83.8
Net -

Net -

Figure 18: Obtained students' net behaviour from Figure 19: Sample outcome of net learning behaviour

Exp-II from our subjects

We can summarize the observations of this paper as follows:

i. As the prime features, both the eyes openness and positive facial postures contribute to determining student's net learning bahaviour.

ii. Through periodical interaction (session-wise corrective measures) by the instructor, students learning behaviour can be maintained or improved in real-time.

\section{Conclusion}

This paper introduces a novel deep ensemble learning-based students' behaviour monitoring framework to analyze the students attention and facial postures in the class room. This real-time feedbacks from the students help the instructor to take the corrective measures either through making changes in the class contents, delivery or individual counseling. Eye openness indicates the wakefulness of a person. It is necessary but not sufficient to conclude whether student 
with fully opened eyes is giving appropriate attention to the lecture or not. That aspect is reinforced by adding facial postures of a student as a measure in determining student's net learning behaviour. Our obtained results help us in corroborating that the eyes openness (we term it as attention) and the facial postures have strong positive correlation. Again, the results suggest that there are similarities in the students learning behaviours between attention and posturebased outcomes for a same session. Thus, our proposed learning behavioral pattern has been further strengthened by combining the attention and the facial postures of a student while attending a lecture.

In future, the proposed approach will be implemented in the real class room scenario to examine its scalability and robustness challenges.

\section{Compliance with ethical standards}

Financial support and funding Nil.

Conflict of interest The authors declare that they have no conflict of interest.

Informed Consent Informed consent was obtained from all individual participants included in the study.

Ethical Approval All procedures performed in studies involving human participants were in accordance with the ethical standards of the institutional and/or national research committee and with the 1964 Helsinki declaration and its later amendments or comparable ethical standards.

\section{References}

[1] Yongchang Li, Xiaowei Li, Martyn Ratcliffe, Li Liu, Yanbing Qi, and Quanying Liu. A real-time eeg-based bci system for attention recognition in ubiquitous environment. In Proceedings of 2011 international workshop on Ubiquitous affective awareness and intelligent interaction, pages 33-40. ACM, 2011.

[2] Angela Riccio, Luca Simione, Francesca Schettini, Alessia Pizzimenti, Maurizio Inghilleri, Marta Olivetti Belardinelli, Donatella Mattia, and Febo Cincotti. Attention and p300-based bci performance in people with amyotrophic lateral sclerosis. Frontiers in human neuroscience, 7:732, 2013.

[3] Rajdeep Chatterjee and Tathagata Bandyopadhyay. Eeg based motor imagery classification using svm and mlp. In 2016 2nd International Conference on Computational Intelligence and Networks (CINE), pages 84-89. IEEE, 2016.

[4] Trevor Darrell and Alex Paul Pentland. Attention-driven expression and gesture analysis in an interactive environment. Vision and Modeling Group, Media Laboratory, Massachusetts Institute of ..., 1995.

[5] Sujit Kumar Gupta, TS Ashwin, and Ram Mohana Reddy Guddeti. Students' affective content analysis in smart classroom environment using deep learning techniques. Multimedia Tools and Applications, pages 1-28, 2019.

[6] Yu-Fei Ma and Hong-Jiang Zhang. Contrast-based image attention analysis by using fuzzy growing. In Proceedings of the eleventh ACM international conference on Multimedia, pages 374-381. ACM, 2003.

[7] DoNALD H ENLow and JAMES A MCNAMARA JR. The neurocranial basis for facial form and pattern. The Angle Orthodontist, 43(3):256-270, 1973.

[8] Caifeng Shan, Shaogang Gong, and Peter W McOwan. Beyond facial expressions: Learning human emotion from body gestures. In $B M V C$, pages 1-10, 2007.

[9] Rawia Bdiwi, Cyril de Runz, Sami Faiz, and Arab Ali Cherif. Smart learning environment: Teacher's role in assessing classroom attention. Research in Learning Technology, 27, 2019.

[10] Feifan Shen, Lingjian Ye, Xiushui Ma, and Weihong Zhong. Smart classroom: An improved smart learning paradigm for college education. In 3rd International Seminar on Education Innovation and Economic Management (SEIEM 2018). Atlantis Press, 2019.

[11] Carrie Anna Courtad. Making your classroom smart: Universal design for learning and technology. In Smart Education and e-Learning 2019, pages 501-510. Springer, 2019.

[12] Xiao-Lei Zhang and DeLiang Wang. A deep ensemble learning method for monaural speech separation. IEEE/ACM Transactions on Audio, Speech and Language Processing (TASLP), 24(5):967-977, 2016. 
[13] Jiewan Zheng, Xianbin Cao, Baochang Zhang, Xiantong Zhen, and Xiangbo Su. Deep ensemble machine for video classification. IEEE transactions on neural networks and learning systems, 30(2):553-565, 2018.

[14] Alex Krizhevsky, Ilya Sutskever, and Geoffrey E Hinton. Imagenet classification with deep convolutional neural networks. In Advances in neural information processing systems, pages 1097-1105, 2012.

[15] Wei Liu, Miaohui Zhang, Zhiming Luo, and Yuanzheng Cai. An ensemble deep learning method for vehicle type classification on visual traffic surveillance sensors. IEEE Access, 5:24417-24425, 2017.

[16] Patrick Lucey, Jeffrey F Cohn, Takeo Kanade, Jason Saragih, Zara Ambadar, and Iain Matthews. The extended cohn-kanade dataset (ck+): A complete dataset for action unit and emotion-specified expression. In 2010 IEEE Computer Society Conference on Computer Vision and Pattern Recognition-Workshops, pages 94-101. IEEE, 2010.

[17] Xiaoyang Tan, Jun Liu, and Songcan Chen. Sub-intrapersonal space analysis for face recognition. Neurocomputing, 69(13-15):1796-1801, 2006.

[18] Fengyi Song, Xiaoyang Tan, and Songcan Chen. Exploiting relationship between attributes for improved face verification. Computer Vision and Image Understanding, 122:143-154, 2014.

[19] Debapriya Maji, Anirban Santara, Pabitra Mitra, and Debdoot Sheet. Ensemble of deep convolutional neural networks for learning to detect retinal vessels in fundus images. arXiv preprint arXiv:1603.04833, 2016. 